\title{
Neuroimaging Findings in Children with Constitutional Mismatch Repair Deficiency Syndrome
}

\author{
(D) A. Kerpel, (D) M. Yalon, (D) M. Soudack, (D). Chiang, (D) A. Gajjar, (D) K.E. Nichols, (D) Z. Patay, (D) S. Shrot, and (D) C. Hoffmann
}

\begin{abstract}
BACKGROUND AND PURPOSE: Constitutional mismatch repair deficiency is a hereditary childhood cancer predisposition syndrome characterized by brain tumors and colorectal and hematologic malignancies. Our objective was to describe the neuroimaging findings in patients with constitutional mismatch repair deficiency.
\end{abstract}

MATERIALS AND METHODS: This retrospective study included 14 children with genetically confirmed constitutional mismatch repair deficiency who were referred to 2 tertiary pediatric oncology centers.

RESULTS: Fourteen patients from 11 different families had diagnosed constitutional mismatch repair deficiency. The mean age at presentation was 9.3 years (range, 5-14 years). The most common clinical presentation was brain malignancy, diagnosed in 13 of the 14 patients. The most common brain tumors were glioblastoma ( $n=7$ patients), anaplastic astrocytoma ( $n=3$ patients), and diffuse astrocytoma ( $n=3$ patients). Nonspecific subcortical white matter T2 hyperintensities were noted in 10 patients (71\%). Subcortical hyperintensities transformed into overt brain tumors on follow-up imaging in 3 patients. Additional non-neoplastic brain MR imaging findings included developmental venous anomalies in 12 patients (85\%) and nontherapy-induced cavernous hemangiomas in 3 patients (21\%).

CONCLUSIONS: On brain MR imaging, these patients have both highly characteristic intra-axial tumors (typically multifocal highgrade gliomas) and nonspecific findings, some of which might represent early stages of neoplastic transformation. The incidence of developmental venous anomalies is high in these patients for unclear reasons. Awareness of these imaging findings, especially in combination, is important to raise the suspicion of constitutional mismatch repair deficiency in routine diagnostic imaging evaluation or surveillance imaging studies of asymptomatic carriers because early identification of the phenotypic "gestalt" might improve outcomes.

ABBREVIATIONS: CMMRD = constitutional mismatch repair deficiency; DVA $=$ developmental venous anomaly; WHO $=$ World Health Organization

D NA replication is a highly controlled process during cell division. The DNA mismatch repair system primarily functions to correct errors arising during DNA replication. Hence, mutations in mismatch repair genes and their dysfunctional protein products lead to accumulation of other "unrepaired" mutations in each cell division, which could cause neoplastic (malignancies, premalignancies, and benign tumors) or non-neoplastic phenotypic features to develop. In humans, germline

Received December 4, 2019; accepted after revision February 26, 2020. From the Department of Radiology (A.K., M.S., S.S., C.H.), Sheba Medical Center, Tel Hashomer, Ramat Gan, Israel; Pediatric Hemato-Oncology (M.Y.), Edmond and Lilly Safra Children's Hospital and Cancer Research Center, Sheba Medical Center, Tel Hashomer, Ramat Gan, Israel; Sackler School of Medicine (A.K., M.Y., M.S., S.S., C.H.), Tel Aviv University, Tel Aviv, Israel; and Department of Pathology (J.C.), Divisions of Neuro-Oncology (A.G.), and Cancer Predisposition (K.E.N.), and Department of Oncology and Section of Neuroimaging, Department of Diagnostic Imaging (Z.P.), St. Jude Children's Research Hospital, Memphis, Tennessee.

A. Kerpel and M. Yalon contributed equally to this article. S. Shrot and C. Hoffmann contributed equally to this article. mutations in the mismatch repair genes result in distinct cancer predisposition syndromes. ${ }^{1-3}$ Heterozygous germline mutations in 1 of the 4 mismatch repair genes (mutL homolog 1 [MLH1], PMS1 homolog 2, mismatch repair system component [PMS2], mutS homolog 2 [MSH2], or mutS homolog 6 [MSH6]) result in Lynch syndrome, which is the most frequent form of inherited colorectal cancer. ${ }^{4}$ Lynch syndrome manifests in early adulthood and is characterized by an increased incidence of endometrial and genitourinary cancer. ${ }^{4,5}$ Biallelic germline mutations in the mismatch repair genes result in a distinct phenotypically

This work was supported, in part, by the American Lebanese Syrian Associated Charities.

Please address correspondence to Ariel Kerpel, MD, Sheba Medical Center, Tel Hashomer, Emek HaEla St 1, Ramat Gan 52621, Israel; e-mail: arikerp@gmail.com

Indicates article with supplemental on-line table.

http://dx.doi.org/10.3174/ajnr.A6512 


\section{Criteria}

Indication for CMMRD testing
Malignancies/premalignancies: 1 is mandatory; if $>1$ is present in the patient, add the points
Carcinoma from the LS spectrum ${ }^{b}$ at younger than 25 years of age
Multiple bowel adenomas at younger than 25 years of age and absence of APC/MUTYH mutations or a single
high-grade dysplasia adenoma at younger than 25 years of age
WHO grade III or IV glioma at younger than 25 years of age
NHL of T-cell lineage or sPNET at younger than 18 years of age
Any malignancy at younger than 18 years of age
Additional features: optional; if $>1$ of the following is present, add the points
Clinical sign of NFl and/or $\geq 2$ hyperpigmented and/or hypopigmented skin alterations $\varnothing>1 \mathrm{~cm}$ in the patient
Diagnosis of LS in a first-degree or second-degree relative
Carcinoma from LS spectrumb before 60 years of age in first-degree, second-degree, and third-degree relatives
A sibling with carcinoma from the LS spectrum, ${ }^{b}$ high-grade glioma, sPNET, or NHL
A sibling with any type of childhood malignancy
Multiple pilomatricomas in the patient
One pilomatricoma in the patient
Agenesis of the corpus callosum or nontherapy-induced cavernoma in the patient
Consanguineous parents
Deficiency/reduced levels of immunoglobulin $(I g) G 2 / 4$ and/or IgA
1 points
1 point
2 points
1 point
1 point
1 point
1 point

Note:-LS indicates Lynch syndrome; NHL, non-Hodgkin lymphoma; sPNET, supratentorial primitive neuroectodermal tumor; NFI, neurofibromatosis type I.

${ }^{a}$ Reprinted with permission from Wimmer et al. ${ }^{5}$

${ }^{b}$ Colorectal, endometrial, small bowel, ureter, renal pelvis, biliary tract, stomach, bladder carcinoma.

defined constitutional mismatch repair deficiency syndrome (CMMRD). ${ }^{2,6-8}$ Individuals with biallelic mismatch repair gene mutations are at risk of developing a vast spectrum of malignancies carrying a dismal prognosis during childhood. Typical malignancies include brain tumors, digestive tract tumors, hematologic malignancies, and Lynch syndrome-associated tumors other than in the digestive tract. ${ }^{2,5,6,9}$ Synchronous and metachronous cancers are common. ${ }^{10,11}$ A proposed scoring system based on clinical features was developed by the European Consortium "Care for CMMRD"5 (Table 1), indicating which patients should be further tested for CMMRD.

Early diagnosis of CMMRD could have considerable clinical importance, not only for surveillance of the index patient but also for screening in the entire pedigree. ${ }^{5}$ CMMRD should be considered in children with multiple malignancies and café au lait spots without the genetic diagnosis of neurofibromatosis type $1 .{ }^{12}$ Remarkably, in children with CMMRD, both parents almost always have Lynch syndrome. Because genetic testing has considerably improved in recent years, our ability to confidently diagnose CMMRD has increased. Surveillance guidelines are also emerging (eg, European Consortium "Care for CMMRD," US Multi-Society Task Force on Colorectal Cancer, ${ }^{4}$ and American Association of Cancer Research). ${ }^{8}$ Recently, a remarkable response to immune checkpoint inhibitors has been reported in patients with CMMRD with glioblastoma. ${ }^{11}$ Hence, early detection of brain tumors and precancerous conditions might offer opportunities to improve outcomes. ${ }^{5,713}$

Our objective is to describe neuroimaging findings in children with both CMMRD and brain tumors, thus increasing neuroradiologists' awareness of this newly discovered cancer predisposition syndrome.

\section{MATERIALS AND METHODS Study Design and Patient Eligibility}

Children with CMMRD diagnosed in Sheba Medical Center, Tel Hashomer, Ramat Gan, Israel and St. Jude Children's Research
Hospital, Memphis, Tennessee between 2014 and 2019 were included in this retrospective study. Review of the patients' medical records provided demographic, clinical, and genetic data. Brain MR imaging studies were reviewed by board-certified neuroradiologists in each participating institution. This retrospective study was approved by the institutional review boards in both hospitals.

\section{Imaging Studies}

MR imaging was performed on the following platforms: $1.5 \mathrm{~T}$ (Magnetom Avanto or Magnetom Essenza, Siemens; Optima MR450w, GE Healthcare) or 3T (Ingenia, Philips Healthcare; Signa, GE Healthcare; Magnetom Prisma or Skyra, Siemens). Routine brain imaging protocols were different at each institution but always included multiplanar nonenhanced T1-weighted images, T2weighted images, diffusion-weighted images, and postcontrast T1weighted sequences, which were occasionally and inconsistently complemented by precontrast T2-FLAIR, SWI/T2*, and postcontrast T2-FLAIR sequences. Acquisition parameters differed according to the specific MR imaging vendors.

\section{Statistical Analysis}

Descriptive statistics were used to analyze data. Continuous variables are presented as mean and range.

\section{RESULTS}

\section{Clinical Data and Tumor Types}

Fourteen patients from 11 families with diagnosed CMMRD were included in this retrospective case series ( 5 boys and 9 girls). A summary of the patients' clinical and genetic features is presented in the On-line Table. Twelve patients (86\%) had MSH6 mutations and 2 (14\%) had PMS2 mutations. Consanguinity was found in 4 of the 11 families. The mean age at diagnosis was 9.3 years (range, 514 years). Multiple synchronous and metachronous malignancies were common. Eighteen brain tumors were found in 13 of 14 patients (93\%). Colorectal adenocarcinoma was diagnosed in 4 
patients (29\%), and 2 patients had multiple colorectal polyps with no high-grade features. Hematologic malignancies were diagnosed in 3 of 14 patients (21\%), 2 with non-Hodgkin lymphomas of the T-cell lineage and 1 with B-cell acute lymphoblastic leukemia. Café au lait spots were observed on physical examination on all patients in our cohort. The mean surveillance period was 4.6 years (range, $1-9$ years).

\section{Neoplastic Neuroimaging Findings}

Glioblastoma (World Health Organization [WHO] IV) was the most common brain malignancy in our cohort and was diagnosed in 7 patients (50\%). All these tumors were supratentorial. On MR imaging, the lesions were irregular, poorly marginated,
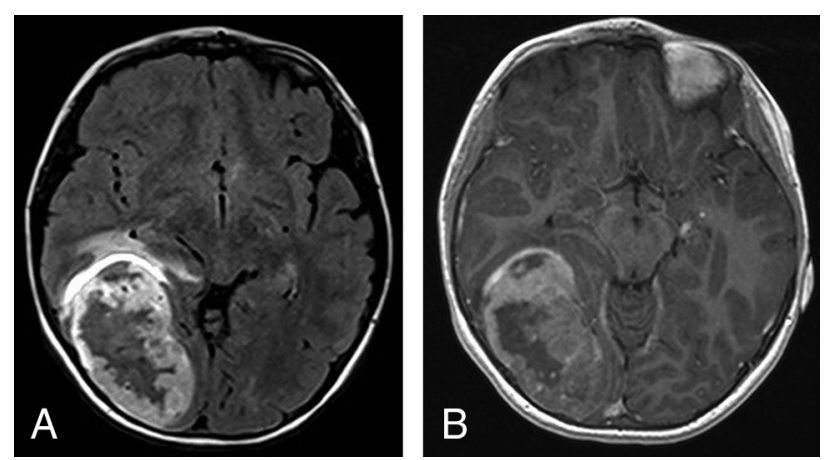

FIG 1. MR imaging of an 8-year-old girl with CMMRD (patient 6). Axial T2-FLAIR $(A)$ and postcontrast $\Pi(B)$ show a right occipitotemporal heterogeneously enhancing mass with regional mass effect. Pathology results indicated glioblastoma.
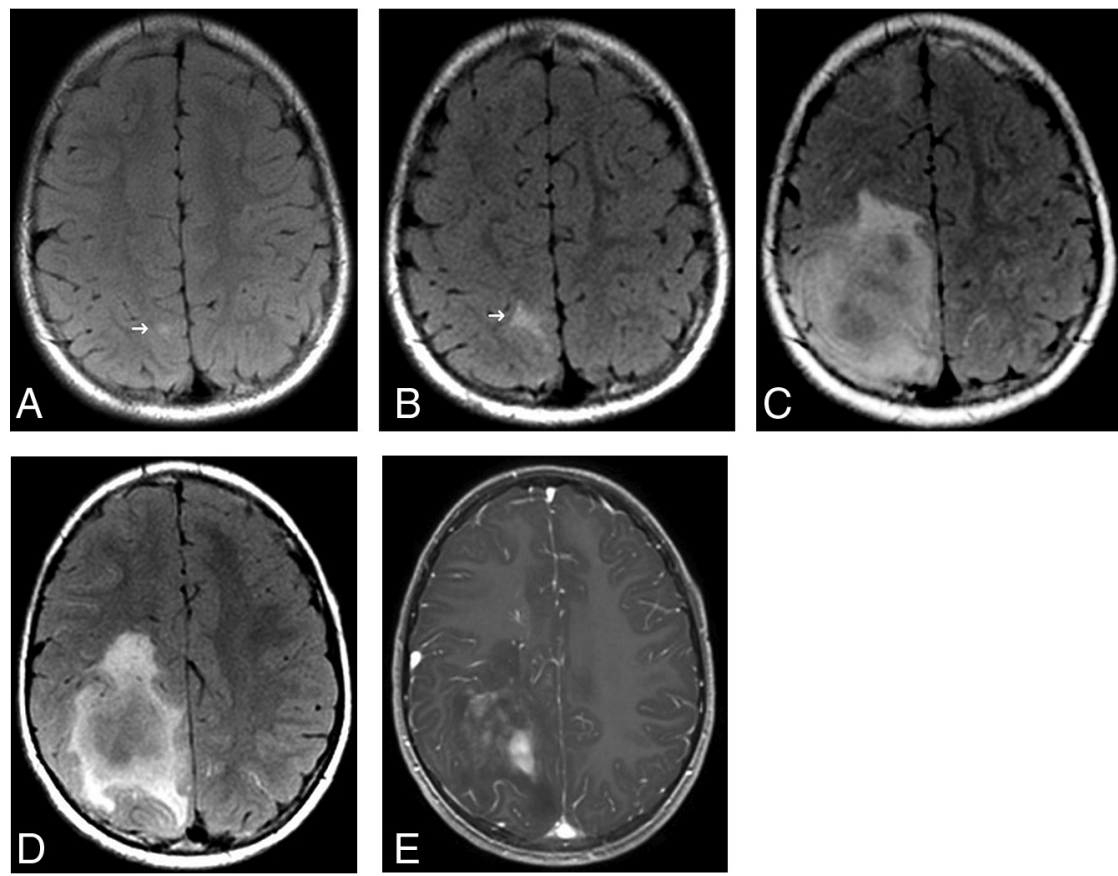

FIG 2. MR images of a boy with CMMRD (patient 3). Routine surveillance imaging at 8 years of age shows subtle right parietal subcortical T2-FLAIR hyperintensity (arrow in A). This subcortical T2-FLAIR hyperintensity increased in size a year later (arrow in B). Two years later, a large heterogeneously enhancing mass is evident on T2-FLAIR ( $C$ and $D$ ) and postcontrast TIWI (E). The histopathologic diagnosis is glioblastoma (WHO IV). and resulted in marked mass effect. Typically, these tumors showed low-T1 and heterogeneous high-T2 or T2-FLAIR signal, with thick irregular peripheral enhancement surrounding central necrosis (Fig 1). The enhancing margins showed some degree of diffusion restriction. In 1 patient, glioblastoma showed irregular patchy areas of enhancement without characteristic central necrosis (Fig 2). Prominent T2 flow voids (ie, prominent feeding vessels) were also noted.

Supratentorial diffuse astrocytoma (WHO II) was diagnosed in 3 patients. On imaging, infiltration and expansion of the overlying cortex were noted. No contrast enhancement or restricted diffusion was identified in these cases. Supratentorial anaplastic astrocytoma (WHO III) was diagnosed in another 3 patients. One tumor of the latter group was partly intraventricular. Diffuse brain stem tumors were found in 2 patients ( 1 with diffuse intrinsic pontine glioma and 1 with glioblastoma). Pleomorphic xanthoastrocytoma (WHO II) involving the temporal lobe was diagnosed in 1 patient. Sonic hedgehog (SHH)-activated medulloblastoma (WHO IV) was diagnosed in 1 patient.

Spinal imaging, performed routinely as part of the surveillance protocol, did not show any structural or neoplastic spinal cord lesions.

\section{Nonspecific, Subcortical T2/T2-FLAIR Hyperintensities}

In our cohort, 10 of 14 patients (71\%) had single or multiple nonspecific patchy subcortical white matter T2-FLAIR hyperintensities, most commonly in the frontal and parietal lobes, but occasionally in the cerebellum and/or the brain stem (Table 2). No mass effect, enhancement, or restricted diffusion were noted in these lesions (Figs 1 to 3). Most of the lesions were stable or showed very modest changes during the surveillance period (Fig 4). In 3 patients, a gradual transformation toward overt brain tumors was observed on surveillance studies (2 with glioblastomas and 1 with diffuse astrocytoma; Figs 2 and 3, respectively). Occasionally, additional new lesions emerged during the follow-up period, too.

\section{Vascular Abnormalities and Focal Areas of Signal Intensity}

Developmental Venous Anomaly. Twelve of the 14 patients (86\%) presented with a network of prominent medullary veins converging into a single draining vein (caput medusae), meeting the conventional MR imaging criteria of developmental venous anomaly (DVA). These DVAs drained into the dural sinuses or ependymal veins. The average number of DVAs in patients who had them was 2.5 (range, 1-5). These DVAs were already present at the initial diagnostic imaging evaluation. DVAs were either supra- (mostly frontoparietal) or infratentorial (cere- 
Table 2: Nonmalignant neuroradiologic findings

\begin{tabular}{|c|c|c|c|c|}
\hline Patient & $\begin{array}{l}\text { T2-FLAIR Subcortical } \\
\text { Hyperintensities }\end{array}$ & $\begin{array}{l}\text { Focal Areas of T2-FLAIR Hyperintense } \\
\text { Signal Intensity (Basal Ganglia, } \\
\text { Thalamus, Pons, or Cerebellum) }\end{array}$ & $\begin{array}{c}\text { Developmental Venous } \\
\text { Anomalies }\end{array}$ & Cavernous Hemangioma \\
\hline 1 & Parietal & - & Cerebellum & Frontal periventricular \\
\hline 2 & - & - & Frontal, cerebellum & - \\
\hline 3 & Frontal & - & Frontal, cerebellum & Pons \\
\hline 4 & - & - & - & - \\
\hline 5 & Frontal, parietal & + & Frontoparietal & - \\
\hline 6 & $\begin{array}{l}\text { Frontal, parietal, } \\
\text { occipital }\end{array}$ & - & Insula & Temporal \\
\hline 7 & - & - & Pons & - \\
\hline 8 & Frontal, temporal & - & Frontal & - \\
\hline 9 & - & - & Frontal, temporal & - \\
\hline 10 & $\begin{array}{l}\text { Frontal, parietal, } \\
\text { temporal, occipital }\end{array}$ & + & Frontal, parietal & - \\
\hline 11 & $\begin{array}{l}\text { Temporal, cerebellar } \\
\text { peduncle }\end{array}$ & + & $\begin{array}{l}\text { Frontal, temporal, } \\
\text { cerebellum }\end{array}$ & - \\
\hline 12 & $\begin{array}{l}\text { Cerebellum, cerebellar } \\
\text { peduncle, frontal } \\
\text { brain stem }\end{array}$ & + & - & - \\
\hline 13 & Frontal, temporal & - & Frontal, temporal & - \\
\hline 14 & Frontal & - & Frontal & - \\
\hline
\end{tabular}

Note:-- indicates absent; +, present.
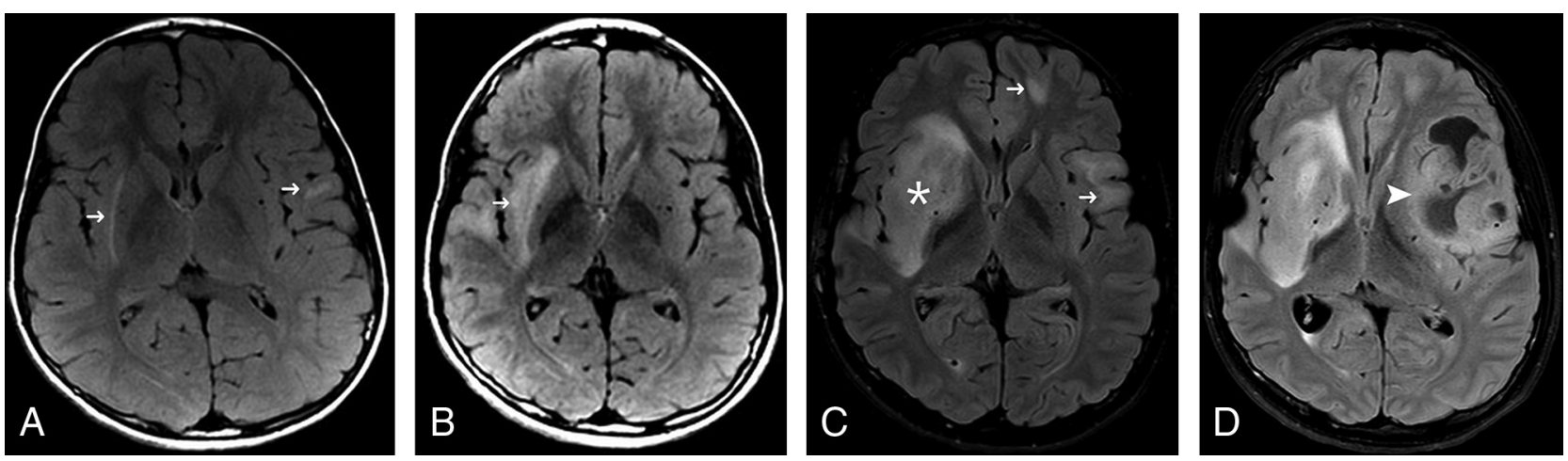

FIG 3. Surveillance brain MR imaging studies of a boy with CMMRD (patient 2). Serial T2-FLAIR imaging shows nonspecific subcortical hyperintensities (arrows) slowly increasing in size (10 years of age, $A$; 15 years of age, $B$ ). At 18 years of age $(C)$, there is an infiltrative lesion involving the right insula (asterisk) and base of the right frontal lobe. Biopsy results indicated diffuse astrocytoma (WHO II). Three months later (D), marked increase in the left frontotemporal lesion was obvious along with central necrosis (arrowhead). This lesion was resected, and pathologic testing indicated glioblastoma.
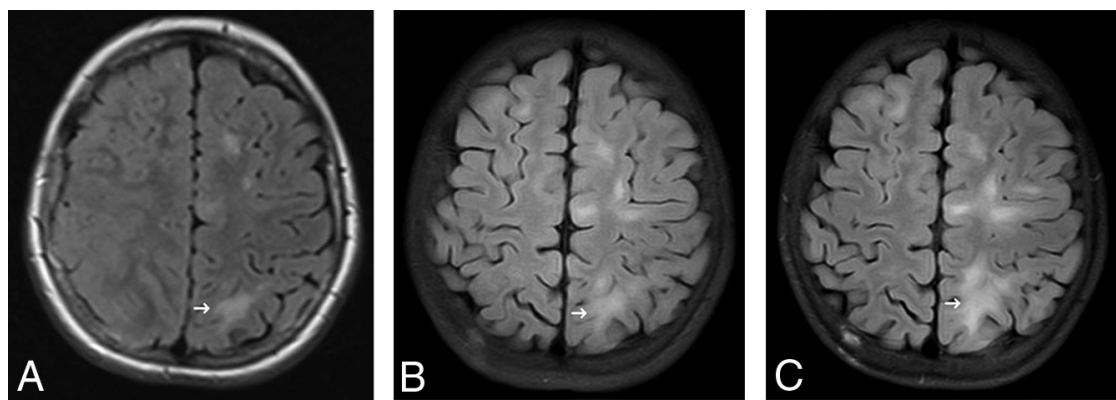

FIG 4. MR images of a girl with CMMRD (patient 6) and glioblastoma (not shown). A gradual increase in frontoparietal subcortical T2-FLAIR hyperintensities (arrows) is noted during 2 years of surveillance ( 7 years of age, $A ; 8$ years of age, $B$; and 9 years of age, $C$ ).

bellum and pons) or in both locations. No spatial association was found between DVAs and other parenchymal abnormalities (overt brain tumors, subcortical T2/T2-FLAIR hyperintensities) (Fig 5).
These findings were seen on initial imaging with no new DVAs identified during follow-up.

Non-Therapy-Related Cavernous Hemangioma. Popcorn-like nonenhancing lesions with prominent $\mathrm{T}^{\star} * /$ SWI blooming artifacts suggestive of cavernous hemangiomas were identified in 3 of 14 patients (21\%). These findings were seen on initial imaging with no new nontherapy-induced cavernomas identified during follow-up. After radiation therapy, several patients showed multiple foci of microhemorrhage, yet these were not considered to be primary cavernous hemangiomas. Not all patients had $\mathrm{T}^{\star}$ or SWI sequences included in the MR imaging protocol. 

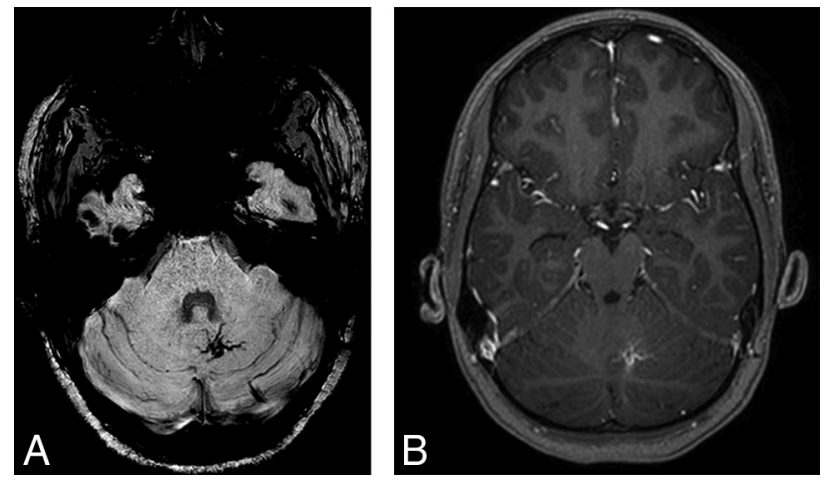

FIG 5. Left cerebellar developmental venous anomaly seen in patient 1 , as shown by axial SWI $(A)$ and postcontrast Tl imaging $(B)$.

Focal Areas of Signal Intensity. Four patients showed nonenhancing T2/T2-FLAIR hyperintense white matter lesions within the supratentorial deep gray matter nuclei (globus pallidus and thalamus), similar to patchy T2/T2-FLAIR hyperintensities commonly seen in neurofibromatosis type 1 (Fig 6). All 4 patients were younger than 15 years of age. All lesions persisted during follow-up.

\section{DISCUSSION}

Here, we report the neuroimaging findings in a series of patients with CMMRD. As previously published, brain tumors, particularly high-grade gliomas, are common in patients with CMMRD. ${ }^{14}$ In children, high-grade gliomas are usually primary, rarely originating from pre-existing low-grade tumors. ${ }^{15}$ Recent data based on molecular abnormalities found in patients with cancer predisposition syndromes suggest that high-grade gliomas in that setting might be secondary (ie, following malignant transformation of low-grade gliomas), ${ }^{16}$ as seen typically in young adult patients. Subcortical T2/ T2-FLAIR hyperintensities described in patients with CMMRD in our study might support these findings because some of the highgrade gliomas eventually emerged from these lesions, possibly as a result of the relentlessly increasing mutation burden during the disease course. Therefore, we hypothesize that accumulation of somatic mutations in patients with CMMRD leads to these lowgrade/precancerous lesions, similar to low-grade and dysplastic polyps found on gastrointestinal endoscopy in patients with CMMRD, which may evolve to malignancies. Unfortunately, histopathologic analysis of these ill-defined subcortical lesions is lacking because no biopsies of those lesions were performed in our patients.

High-grade glioma was the most prevalent brain tumor in our patients with CMMRD, as in previous reports. ${ }^{14}$ Nevertheless, low-grade gliomas were not uncommon (found in $21 \%$ of our patient cohort). Supratentorial embryonal tumors, which have been reported previously ${ }^{17}$ in patients with CMMRD, were not found in our series. The median age of our patients at diagnosis of brain tumors was 9.3 years (range, 5-14 years of age), as has been previously reported. ${ }^{17}$ The imaging characteristics of various brain tumors in our series resembled those in brain tumors in patients without CMMRD. Nevertheless, recent genetic evaluation of pediatric brain tumors shows that in patients with CMMRD, mutations in mismatch repair genes may lead to

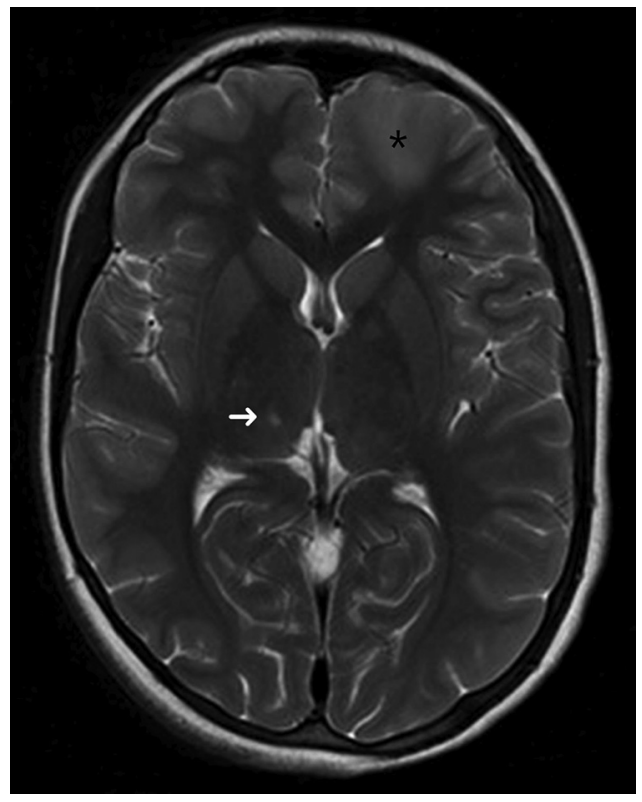

FIG 6. T2-weighted image of a diffuse astrocytoma in the left frontal lobe (asterisk) of a 14-year-old girl (patient 5). Ovoid nonenhancing hyperintense foci are noted in the right thalamus (arrow) and left globus pallidus, similar to focal areas of signal intensities commonly seen in patients with neurofibromatosis type 1 .

accumulation of multiple somatic mutations, resulting in considerably higher tumoral mutation burden than that in nonCMMRD childhood gliomas. ${ }^{18}$

In humans, most CMMRD cases are due to MSH6 or PMS2 mutation. Similarly, in our cohort, 12 patients had MSH6 mutations $(86 \%)$ and $2(14 \%)$ had PMS2 mutations. Individuals with these mutations in the context of Lynch syndrome exhibit low penetrance, which might partially explain the negative family history in our cohort. ${ }^{4,12}$ Additionally, patients with PMS2 and MSH6 mutations have a higher reported prevalence of brain tumors than do those with other mismatch repair mutations, and these patients are more likely to survive their first tumor and develop a metachronous tumor. ${ }^{2}$

Individuals with CMMRD frequently display neurofibromatosis type 1 stigmata, such as café au lait spots. All the patients in our cohort presented with café au lait spots. Brain lesions similar to the focal areas of signal intensity, commonly seen in prepubertal patients with neurofibromatosis type 1, were identified in 4 of our patients. All 4 showed lesions before 15 years of age, in a timeline similar to that of neurofibromatosis type $1,{ }^{19}$ yet all persisted during the available follow-up. The associations between CMMRD and neurofibromatosis type 1 have been described by Wimmer et al, ${ }^{3}$ and a presumed explanation is that common somatic mutations in the neurofibromin 1 (NF1) gene are acquired as a consequence of the mismatch repair malfunction.

Structural brain abnormalities were reported by Baas et $\mathrm{al}^{20}$ in a case series of 4 patients with CMMRD with agenesis of the corpus callosum and gray matter heterotopia. Such malformative abnormalities were not found in our cohort.

Additional non-neoplastic brain MR imaging findings in CMMRD include DVAs and nontherapy-induced cavernous hemangiomas, as have been recently reported. ${ }^{20,21}$ Remarkably, 12 of 
our 14 patients had DVAs (typically multiple DVAs), indicating a considerably higher prevalence than that reported in healthy populations $(6 \%-7 \%){ }^{22}$ Because DVAs are considered a normal variant, under-reporting is probably common. However, patients with primary brain tumors have a higher prevalence of DVAs than the healthy population ( $10.2 \%$ versus $5.3 \%$, respectively). ${ }^{23}$ In our series of children with brain tumors and CMMRD, there was no apparent association between the tumor location and the location of the DVA. DVAs have a well-recognized association with cavernomas, ${ }^{24}$ which was also noted in some of our patients. The pathogenesis of these vascular anomalies is not clearly understood. DVAs might arise as a response to compromise of the normal venous drainage by stenosis, thrombosis, or maldevelopment. Cavernomas might occur in both sporadic and familial forms. Patients with familial cavernomas typically have multiple malformations. Familial cavernomas have been linked to 3 specific cerebral cavernous malformation genes KRIT1/CCM1 (Krev interaction trapped 1), MGC4607/CCM2 (cerebral cavernous malformation 2), or PDCD10/CCM3 (programmed cell death protein 10). Almost $80 \%$ of patients with the hereditary form have a heterozygous germline mutation in 1 of these 3 genes. Development of sporadic cavernomas is thought to be secondary to microhemorrhages and angiogenic growth factor activation in a causeand-effect relationship with adjacent DVA. ${ }^{23,25,26}$ Recognizing vascular abnormalities in the brain, particularly DVAs, is important; hence, they seem to contribute to a potentially suggestive "imaging gestalt" in children with CMMRD.

CMMRD should be clinically suspected in children with brain tumors who have café au lait spots, a family history of Lynch syndrome, or a sibling with childhood cancer manifesting as brain tumors or lymphoproliferative malignancies. Because high-grade gliomas are uncommon in neurofibromatosis type 1, any child with a clinical suspicion of this syndrome and a high-grade tumor should also be evaluated for CMMRD. ${ }^{17}$ Because it is an autosomal recessive syndrome, consanguinity is a common feature, as seen in 3 of the families included in our study. The final confirmation of the diagnosis of CMMRD should come from the determination of the causative biallelic mutations of the patient.

Because penetrance is high, reaching $>90 \%$ at 20 years of age and almost all patients develop cancer as children, surveillance is crucial. Consensus surveillance protocols were recently published, which advocate brain MR imaging every 6 months following diagnosis. ${ }^{8}$ In addition to screening for brain tumors, close attention to nonspecific subcortical T2/T2-FLAIR hyperintensities is important, as shown in the current study, because those lesions have the potential for malignant neoplastic transformation with time. Nuclear medicine studies including ${ }^{11} \mathrm{C}$ methionine PET and ${ }^{18}$ F-fluoroethyl-L-tyrosine PET have increased sensitivity to detect insidiously developing brain tumors. ${ }^{27}$ This was also demonstrated in another hereditary cancer predisposition syndrome, L-2-hydroxyglutaric acidu$\mathrm{ria}^{28}$ hence, it could be a useful adjunct to any MR imaging study, raising the possibility of neoplastic transformation of the subcortical T2 hyperintensities. Additional surveillance imaging studies include annual whole-body MR imaging beginning at 6 years of age (this should not replace a dedicated brain MR imaging protocol), annual abdominal sonography beginning at
1 year of age, and annual transvaginal sonography beginning at 20 years of age. ${ }^{8}$

The principal limitation of this study is the relatively small number of patients in our cohort. However, the frequency of the cardinal MR imaging findings, such as cerebral neoplasms, nonspecific subcortical T2 hyperintensities, DVAs, cavernous angiomas, and focal areas of signal intensity, is quite compelling. Further assessment of larger patient cohorts is needed to define and describe the full spectrum of neuroimaging phenotypic features of CMMRD. Additionally, advanced imaging techniques (diffusion- and perfusion-weighted imaging, spectroscopy, and so forth) and/or nuclear medicine (PET) studies are needed to improve our understanding of the natural course of potentially precancerous nonspecific subcortical white matter signal abnormalities for further characterization. Likewise, another limitation is the lack of histopathologic correlation of the nonspecific subcortical T2/T2-FLAIR hyperintensities.

\section{CONCLUSIONS}

On brain imaging, patients with CMMRD present with overt tumors and several nonspecific findings, some of which might be precancerous; however, others, including non-neoplastic vascular anomalies, may also contribute to a suggestive imaging "gestalt." Awareness of these imaging findings is important at both the initial diagnostic imaging evaluation of children with suspected brain tumors and at that of those known to carry the genetic predisposition and requiring regular surveillance studies because early detection of brain neoplasms may improve clinical outcomes.

Disclosures: Amar Gajjar-UNRELATED: Consultancy: Roche Pharmaceuticals. Kim E. Nichols-UNRELATED: Grants/Grants Pending: Incyte, Comments: small research grant to support work ongoing in basic laboratory.

\section{REFERENCES}

1. Jiricny J. The multifaceted mismatch-repair system. Nat Rev Mol Cell Biol 2006;7:335-46 CrossRef Medline

2. Wimmer K, Kratz CP. Constitutional mismatch repair-deficiency syndrome. Haematologica 2010;95:699-701 CrossRef Medline

3. Wimmer K, Rosenbaum T, Messiaen L. Connections between constitutional mismatch repair deficiency syndrome and neurofibromatosis type 1. Clin Genet 2017;91:507-19 CrossRef Medline

4. Durno C, Boland CR, Cohen S, et al. Recommendations on Surveillance and Management of Biallelic Mismatch Repair Deficiency (BMMRD) Syndrome: A Consensus Statement by the US Multi-Society Task Force on Colorectal Cancer. Gastroenterology 2017;152:1605-14 CrossRef Medline

5. Wimmer K, Kratz CP, Vasen HF, et al; EU-Consortium Care for CMMRD (C4CMMRD). Diagnostic criteria for constitutional mismatch repair deficiency syndrome: suggestions of the European Consortium "Care for CMMRD” (C4CMMRD). J Med Genet 2014; 51:355-65 CrossRef Medline

6. Vasen HF, Ghorbanoghi Z, Bourdeaut F, et al; EU-Consortium Care for CMMR-D (C4CMMR-D). Guidelines for surveillance of individuals with constitutional mismatch repair-deficiency proposed by the European Consortium "Care for CMMR-D” (C4CMMR-D). J Med Genet 2014;51:283-93 CrossRef Medline

7. Johannesma PC, van der Klift HM, van Grieken NC, et al. Childhood brain tumours due to germline bi-allelic mismatch repair gene mutations. Clin Genet 2011;80:243-55 CrossRef Medline

8. Tabori U, Hansford JR, Achatz MI, et al. Clinical management and tumor surveillance recommendations of inherited mismatch 
repair deficiency in childhood. Clin Cancer Res 2017;23:e32-37 CrossRef Medline

9. Aronson M, Gallinger S, Cohen Z, et al. Gastrointestinal findings in the largest series of patients with hereditary biallelic mismatch repair deficiency syndrome: report from the international consortium. Am J Gastroenterol 2016;111:275-84 CrossRef Medline

10. Kim B, Tabori U, Hawkins C. An update on the CNS manifestations of brain tumor polyposis syndromes. Acta Neuropathol 2020 Jan 22. [Epub ahead of print] CrossRef Medline

11. Bouffet $\mathrm{E}$, Larouche V, Campbell BB, et al. Immune checkpoint inhibition for hypermutant glioblastoma multiforme resulting from germline biallelic mismatch repair deficiency. J Clin Oncol 2016;34:2206-01 CrossRef Medline

12. Q Rana H, Syngal S. Biallelic mismatch repair deficiency: management and prevention of a devastating manifestation of the Lynch syndrome. Gastroenterology 2017;152:1254-57 CrossRef Medline

13. Shinagare AB, Giardino AA, Jagannathan JP, et al. Hereditary cancer syndromes: a radiologist's perspective. AJR Am J Roentgenol 2011; 197:W1001-07 CrossRef Medline

14. Bakry D, Aronson M, Durno C, et al. Genetic and clinical determinants of constitutional mismatch repair deficiency syndrome: report from the Constitutional Mismatch Repair Deficiency Consortium. Eur J Cancer 2014;50:987-96 CrossRef Medline

15. Broniscer A, Baker SJ, West AN, et al. Clinical and molecular characteristics of malignant transformation of low-grade glioma in children. J Clin Oncol 2007;25:682-89 CrossRef Medline

16. Paugh BS, Qu C, Jones $\mathrm{C}$, et al. Integrated molecular genetic profiling of pediatric high-grade gliomas reveals key differences with the adult disease. J Clin Oncol 2010;28:3061-68 CrossRef Medline

17. Michaeli O, Tabori U. Pediatric high-grade gliomas in the context of cancer predisposition syndromes. J Korean Neurosurg Soc 2018;61:319-332.CrossRef Medline

18. Shlien A, Campbell BB, de Borja R, et al; Biallelic Mismatch Repair Deficiency Consortium. Combined hereditary and somatic mutations of replication error repair genes result in rapid onset of ultra-hypermutated cancers. Nat Genet 2015;47:257-62 CrossRef Medline

19. Itoh T, Magnaldi S, White RM, et al. Neurofibromatosis type 1: the evolution of deep gray and white matter MR abnormalities. AJNR Am J Neuroradiol 1994;15:1513-19 Medline

20. Baas AF, Gabbett M, Rimac M, et al. Agenesis of the corpus callosum and gray matter heterotopia in three patients with constitutional mismatch repair deficiency syndrome. Eur J Hum Genet 2013;21:5561 CrossRef Medline

21. Shiran SI, Ben-Sira L, Elhasid R, et al. Multiple brain developmental venous anomalies as a marker for constitutional mismatch repair deficiency syndrome. AJNR Am J Neuroradiol 2018;39:1943-46 CrossRef Medline

22. Gökçe E, Acu B, Beyhan M, et al. Magnetic resonance imaging findings of developmental venous anomalies. Clin Neuroradiol 2014;24:135-43 CrossRef Medline

23. Jones BV, Linscott L, Koberlein G, et al. Increased prevalence of developmental venous anomalies in children with intracranial neoplasms. AJNR Am J Neuroradiol 2015;36:1782-85 CrossRef Medline

24. Meng G, Bai C, Yu T, et al. The association between cerebral developmental venous anomaly and concomitant cavernous malformation: an observational study using magnetic resonance imaging. BMC Neurol 2014;14:50 CrossRef Medline

25. Riant F, Bergametti F, Ayrignac X, et al. Recent insights into cerebral cavernous malformations: the molecular genetics of CCM. FEBS $J$ 2010;277:1070-75 CrossRef Medline

26. Linscott LL, Leach JL, Jones BV, et al. Developmental venous anomalies of the brain in children - imaging spectrum and update. Pediatr Radiology 2016;46:394-406, quiz 391-93 CrossRef Medline

27. Treglia G, Muoio B, Trevisi G, et al. Diagnostic performance and prognostic value of PET/CT with different tracers for brain tumors: a systematic review of published meta-analyses. Int J Mol Sci 2019;20 CrossRef Medline

28. Patay Z, Orr BA, Shulkin BL, et al. Successive distinct high-grade gliomas in L-2-hydroxyglutaric aciduria. $J$ Inherit Metab Dis 2015;38:273-77 CrossRef Medline 